Proceeding Series of the Brazilian Society of Computational and Applied Mathematics

\title{
Otimização de problemas integrados na indústria papeleira
}

\section{Sônia Cristina Poltroniere Silva}

Faculdade de Ciências, UNESP, Bauru, SP,

Departamento de Matemática

\section{Silvio Alexandre de Araujo ${ }^{2}$}

Instituto de Biociências, Letras e Ciências Exatas, UNESP, São José do Rio Preto, SP, Departamento de Matemática Aplicada

\section{Kelly Cristina Poldi ${ }^{3}$}

Instituto de Matemática, Estatística e Computação Científica, UNICAMP, Campinas, SP.

\begin{abstract}
Resumo. Dois importantes problemas de otimização matemática ocorrem no planejamento e programação da produção em indústrias papeleiras: o problema de dimensionamento de lotes e o problema de corte de estoque multiperíodo. O problema de dimensionamento de lotes deve determinar a quantidade de bobinas jumbos de diferentes tipos de papel a serem produzidos em cada máquina, ao longo de um horizonte de planejamento finito. Estes jumbos são então cortados para atender a demanda de itens para cada período. Neste trabalho, tratamos da integração desses dois problemas, procurando minimizar custos com produção e estoque dos jumbos, como também a perda de papel durante o processo de corte. Diferentes modelagens para o problema integrado são consideradas, e os modelos foram resolvidos heuristicamente usando um pacote de otimização. Procurando obter limitantes inferiores para o problema, foram resolvidas versões relaxadas dos modelos. Resultados computacionais são discutidos.
\end{abstract}

Palavras-chave. Problema de corte de estoque, problema de dimensionamento de lotes, problemas integrados, métodos computacionais.

\section{Introdução}

O problema de corte de estoque, que surge em indústrias de manufatura, consiste na otimização do processo de corte de unidades maiores (objetos) que estejam disponíveis, para a produção de um conjunto de unidades menores (itens), com o objetivo de atender a demanda desses itens e satisfazer algum critério de otimização, por exemplo, minimizar a perda de material gerada pelo corte. Tais problemas vêm sendo investigados nas últimas

\footnotetext{
1 soniacps@fc.unesp.br

2 saraujo@ibilce.unesp.br

${ }^{3}$ kelly.poldi@gmail.com
} 
décadas, desde os trabalhos pioneiros de Gilmore e Gomory (1961, 1963, 1965), que propuseram a técnica de geração de colunas, viabilizando a resolução de problemas reais.

Atualmente, a integração do problema de corte de estoque com outros problemas que ocorrem no planejamento e programação da produção em indústrias tem gerado pesquisas em todo o mundo. Um exemplo é a integração com o problema de dimensionamento de lotes (Trigeiro et al. 1989), que consiste em determinar a quantidade de produtos a serem produzidos em cada período ao longo de um horizonte de tempo finito, de modo a atender certa demanda e otimizar uma função objetivo, como por exemplo, minimizar custos, estoques e atrasos. Em geral, o problema de dimensionamento de lotes (PDL) e o problema de corte de estoque (PCE) são tratados separadamente de forma sequencial, ou seja, primeiro resolve-se o PDL e posteriormente o PCE. No entanto, esta abordagem pode elevar os custos globais. A seguir, são apresentados alguns trabalhos que consideram problemas integrados em contextos industriais.

Farley (1988) foi o primeiro autor a publicar um estudo sobre o PCE integrado ao problema de planejamento e programação da produção, com aplicações na indústria de roupa. Na indústria de papel, podemos citar Respício e Captivo (2002) e Correia et al. (2004). Também com aplicações na indústria de papel, Poltroniere et al. (2008) propuseram um modelo matemático para tratar o PDL e o PCE de forma integrada, num horizonte de planejamento dividido em períodos. Foram propostos dois métodos heurísticos para a solução, baseados na relaxação Lagrangiana das restrições de integração, que se mostraram apropriados para tratar o problema integrado. Considerando a indústria moveleira, Gramani e França (2006) também estudaram a integração do PDL e do PCE . Foram propostos um modelo matemático e um método de solução baseado no problema de caminho mínimo. Gramani et al. $(2009,2010)$ realizaram extensões do modelo proposto em 2006 e novas abordagens de solução. Também podemos citar os trabalhos de Ghidini (2008), Santos et al. (2011) e Vanzela et al. (2012). Recentemente, temos os trabalhos de Silva et al. (2014) e Kallrath et al. (2014).

Em Poltroniere et al. (2008), são obtidas soluções factíveis para o problema integrado. No entanto, não se pode afirmar a qualidade destas soluções, pois não se conhecem as soluções ótimas e nem tampouco limitante inferiores (duais) para o problema. O objetivo deste trabalho consiste na resolução de diferentes modelos matemáticos para o problema proposto em Poltroniere et al. (2008). Além do modelo proposto pelos autores, que considera a abordagem de Gilmore e Gomory (1963) para o problema de corte de estoque, neste trabalho, consideramos também a abordagem de Kantorovich (1960) e de Valério de Carvalho (1999, 2002), que é baseada num modelo de fluxo em redes. Os modelos são implementados usando um pacote de otimização, resolvidos de forma heurística e os resultados são comparados com os obtidos em Poltroniere et al. (2008). Além disso, algumas relaxações são resolvidas com o intuito de obter limitantes inferiores para o problema integrado, o que permitirá testar a qualidade das soluções heurísticas.

\section{Modelos matemáticos}

Poltroniere et al. (2008) propõem um modelo matemático inteiro misto para tratar o PDL e o PCE de forma integrada, num horizonte de planejamento dividido em períodos. O PCE foi modelado usando a abordagem de Gilmore e Gomory (1963), supondo diferentes bobinas em estoque, em quantidades limitadas, como está apresentado na seção 2.1. 


\subsection{Modelo Integrado usando a abordagem de Gilmore e Gomory (LCGG)}

Índices:

$t=1, \ldots, T:$ número de períodos no horizonte de planejamento;

$k=1, \ldots, K:$ número de gramaturas;

$m=1, \ldots, M$ : número de máquinas (máquina $m$ produz bobinas-mestre de largura $L_{m}$ );

$j=1, \ldots, N_{m}$ : número de padrões de corte para as bobinas-mestre do tipo $m$;

$i=1, \ldots, N f:$ número de diferentes tipos de itens demandados;

$\{1, \ldots, N f\}=S(1) \cup S(2) \cup \ldots \cup S(K)$, em que $S(k)=\{i$ tal que o item $i$ tem gramatura $k\}$.

Parâmetros:

$c_{k m t}$ : custo de produção da bobina-mestre de gramatura $k$ na máquina $m$ no período $t$;

$h_{k t}:$ custo/ton de estocar bobinas-mestre de gramatura $k$ no período $t$;

$s_{k m t}$ : custo de preparação da máquina $m$ para produzir bobina-mestre de gram. $k$, período $t$;

$c p_{k t}$ : custo/cm de perda de papel de gramatura $k$ durante o processo de corte no período $t$;

$\sigma_{i t}:$ custo/ton de estocar itens finais do tipo $i$ no período $t$.

$C_{m t}$ : capacidade (ton) da máquina $m$ no período $t$;

$\mathbf{d}_{k t}$ : vetor da demanda de itens finais de gramatura $k$ no período $t$.

$\rho_{k}$ : peso específico da bobina-mestre de gramatura $k$;

$\boldsymbol{\eta}_{k} \quad$ : vetor de pesos dos itens finais de gram. $k$;

$D_{k t}$ : demanda (ton) de papel da gramatura $k$ no período $t\left(D_{k t}=\boldsymbol{\eta}_{k} \mathbf{d}_{k t}\right)$;

$b_{k m}$ : peso da bobina-mestre de gramatura $k$ produzida na máquina $m\left(b_{k m}=L_{m} \rho_{k}\right)$;

$f_{k m}$ : peso do papel desperdiçado na preparação da máquina $m$ para a bobina de gramatura $k$;

$\mathbf{a}_{j m}$ : vetor associado ao padrão de corte $j$ para a bobina-mestre de largura $L_{m}$;

$p_{j m}:$ perda de papel (cm) no padrão de corte $j$ usado na bobina-mestre de largura $L_{m}$;

$Q \quad$ : número grande.

Variáveis de decisão:

$x_{k m t}:$ número de bobinas-mestre de gramatura $k$, produzidas na máquina $m$ no período $t$;

$w_{k m t}$ : estoque de bobinas-mestre de gramatura $k$, máquina $m$ estocadas no fim do período $t$;

$z_{k m t}$ : indicam a produção ou não da bobina-mestre de gram. $k$ na máquina $m$ no período $t$.

$y_{k m t}^{j}$ : número de bobinas-mestre de gramatura $k$ produzidas na máquina $m$ no período $t$, cortadas usando o padrão de corte $j$;

$\mathbf{e}_{k t}$ : vetor de itens finais de gramatura $k$ estocados no final do período $t$.

No modelo a seguir, o parâmetro $D_{k t}$ não é um dado do problema, pois depende da perda que ocorre durante $\mathrm{o}$ processo de corte. Por definição, ele deve ser $D_{k t}=\sum_{i \in S(k)} \eta_{i k} d_{i k t}+$ perda. Destacam-se as restrições (6), que limitam o número de 
bobinas-mestre cortadas àquelas que foram produzidas anteriormente, pois são as restrições de integração que envolvem decisões relativas à produção e ao corte de jumbos.

Modelo matemático LCGG-Lote Corte Gilmore Gomory:

$$
\begin{array}{lll}
\text { Minimizar } & \sum_{t=1}^{T} \sum_{m=1}^{M} \sum_{k=1}^{K}\left(c_{k m t} x_{k m t}+h_{k t} b_{k m} w_{k m t}+s_{k m t} z_{k m t}\right)+\sum_{t=1}^{T} \sum_{k=1}^{K} c p_{k t} F(k, t)+ \\
& +\sum_{t=1}^{T} \sum_{k=1}^{K} \sum_{i \in S(k)} \sigma_{i t} \eta_{i k} e_{i k t} & \\
\text { s. a. } \quad & \sum_{m=1}^{M}\left(b_{k m} x_{k m t}+b_{k m} w_{k, m, t-1}-b_{k m} w_{k m t}\right)=D_{k t}, & k=1, \ldots, K ; t=1, \ldots, T \\
& \sum_{k=1}^{K}\left(b_{k m} x_{k m t}+f_{k m} z_{k m t}\right) \leq C_{m t}, & \\
& x_{k m t} \leq Q \cdot z_{k m t}, & k=1, \ldots, T ; t=1, \ldots, T \\
\sum_{m=1}^{M} \sum_{j=1}^{N_{m}} \mathbf{a}_{j m} y_{k m t}^{j}+\mathbf{e}_{k, t-1}-\mathbf{e}_{k t}=\mathbf{d}_{k t}, K ; m=1, \ldots, T ; t=1, \ldots, T \\
\sum_{j=1}^{N_{m}} y_{k m t}^{j}=x_{k m t}+w_{k, m, t-1}-w_{k m t}, & k=1, \ldots, K ; t=1, \ldots, T \\
w_{k m 0}=0, \mathbf{e}_{k 0}=0, & k=1, \ldots, K ; m=1, \ldots, T ; t=1, \ldots, T \\
x_{k m t} \geq 0, w_{k m t} \geq 0 \text { e inteiros, } & k=1, \ldots, K ; m=1, \ldots, M ; t=1, \ldots, T \\
z_{k m t} \in\{0,1\}, & k=1, \ldots, K ; m=1, \ldots, M ; t=1, \ldots, T \\
y_{k m t}^{j} \geq 0 \text { inteiros, } \mathbf{e}_{k t} \geq 0, \quad j=1, \ldots, N_{m} ; & k=1, \ldots, K ; m=1, \ldots, M ; t=1, \ldots, T
\end{array}
$$

\subsection{Outras abordagens para o Modelo Integrado}

Kantorovich (1960) introduziu uma formulação matemática para o problema de corte de estoque, supondo apenas um tipo de objeto em estoque de largura $L$, em quantidade limitada, com o objetivo de minimizar o número de objetos (bobinas no caso específico da indústria de papel) cortados para atender a uma demanda.

Valério de Carvalho (1999) propôs uma formulação alternativa para o PCE, considerando objetos idênticos em estoque. Trata-se de um problema de fluxo mínimo com restrições adicionais para a satisfação da demanda. Segundo o autor, o modelo linear, obtido pela relaxação das variáveis de integralidade, é equivalente ao modelo de Gilmore e Gomory (1961) e, portanto, os limitantes inferiores em ambos os casos são iguais. A extensão desse modelo para vários tipos de objetos em estoque é proposta em Valério (2002).

Neste trabalho, modelamos o problema integrado proposto em Poltroniere et al. (2008) usando as abordagens de Kantorovich (modelo LCKT) e de Valério de Carvalho (modelo LCVC). Considerando o modelo (1)-(10), na seção 2.1, a função objetivo e os conjuntos de restrições (5), (6) e (10) foram alterados de maneira adequada para cada modelagem proposta pelos autores. 


\section{Heurísticas propostas em Poltroniere et al. (2008)}

Para resolver o problema integrado (1)-(10), Poltroniere et al. (2008) propuseram dois métodos, denominados Heurísticas Lote/Corte e Corte/Lote, baseadas na relaxação lagrangiana das restrições de integração (6) que são adicionadas à função objetivo (1), ponderadas pelas variáveis duais $\gamma_{k m t}$. Assim, o problema (1)-(10) é decomposto em dois subproblemas: PDL e PCE, que são abordados separadamente e iterativamente.

Para resolver o PDL definido pelas restrições (2)-(4), (8) e (9), foi realizada uma adaptação do método proposto por Toledo (1998). A solução obtida supre o PCE com os jumbos a serem cortados. O PCE multiperíodo (restrições (5) acopladas por causa do estoque de itens finais $e_{i k t}$ ) é composto por $K$ subproblemas independentes, um para cada gramatura. Esses $K$ subproblemas foram resolvidos num horizonte dividido em períodos, usando método simplex com geração de colunas proposto por Gilmore e Gomory (1961, 1963) e considerando a condição de integralidade relaxada. Para possibilitar a antecipação de itens durante o processo de corte, utilizamos uma abordagem heurística, proposta em Poltroniere (2006), permitindo novas combinações de itens.

\section{Resultados computacionais}

Foram utilizados os exemplares gerados em Poltroniere et al. (2008). São 27 classes, cada uma delas contendo 10 exemplares, classificados de acordo com o número de itens demandados ( $N f=5,10,20)$, o número de gramaturas $(K=2,4,6)$ e o número de períodos $T=8,10,12$. As bobinas mestre de larguras $L_{1}=540 \mathrm{~cm}$ e $L_{2}=460 \mathrm{~cm}$ e com peso específico $\rho_{k}=2 \mathrm{~kg} / \mathrm{cm}$. Os parâmetros do modelo foram gerados simulando situações encontradas na indústria de papel.

\subsection{Soluções heurísticas}

Os resultados heurísticos obtidos com a resolução dos três modelos, que usam as abordagens de Gilmore e Gomory (LCGG), Kantorovich (LCKT) e Valério de Carvalho (LCVC), utilizando o pacote computacional Cplex 12.6, foram comparados com os resultados apresentados por uma das heurísticas desenvolvidas em Poltroniere et al. (2008), chamada heurística Lote/Corte, como descrita resumidamente na seção 3.

Para a resolução dos modelos LCKT e LCVC, as variáveis do modelo original foram consideradas como inteiras, e o tempo computacional foi limitado em 600 segundos na tentativa de obter uma solução exata. No modelo LCGG, primeiramente, é resolvida a relaxação linear de forma ótima, considerando inteiras as variáveis relativas ao problema da mochila. Posteriormente, considerando somente as colunas da solução ótima da relaxação linear, é dado um limite de 600 segundos para que o pacote encontre uma solução inteira.

Destacam-se os resultados obtidos ao se resolver o modelo LCGG, pois, num tempo computacional relativamente baixo, encontrou-se soluções muitas vezes melhores do que as obtidas pela heurística de Poltroniere et al. (2008). O modelo LCKT não consegue provar a otimalidade para nenhum exemplo, mas encontra soluções factíveis para (somente) 6 exemplares, dentro do tempo disponível. Já o modelo LCVC não conseguiu encontrar soluções factíveis em 600 segundos. 


\subsection{Relaxações}

Versões relaxadas dos modelos apresentados na Seção 2 também foram resolvidas com o objetivo de prover limitantes inferiores para os exemplos e consequentemente ter um parâmetro de teste para a qualidade das heurísticas.

Relaxação 1: as variáveis do problema foram relaxadas, inclusive as variáveis de preparação de máquina (setup), que passaram a ser consideradas contínuas entre 0 e 1 , e as relativas aos padrões de corte. No entanto, as relativas ao problema da mochila foram mantidas como inteiras. Destacam-se aqui a qualidade dos limitantes inferiores obtidos pelo modelo LCGG, que foram os melhores obtidos pelas relaxações.

Relaxação 2: com o intuito de obter limitantes inferiores ainda mais apertados, utilizouse os modelos LCKT e LCVC para resolver o problema com as variáveis de preparação binárias e as restantes contínuas (inclusive as variáveis relativas ao problema da mochila). Observa-se que neste caso não faz sentido a aplicação do modelo LCGG, pois, seria uma solução heurística já que nem todas as colunas estariam disponíveis no processo de arredondamento. Além disso, só é possível garantir que se tem um limitante inferior, quando os modelos LCKT e LCVC resolvem o problema de forma ótima, o que ocorreu para todas as instâncias, num tempo computacional bastante baixo. Neste caso, destacam-se como melhores, os limitantes obtidos pelo modelo LCVC.

\section{Conclusões e perspectivas futuras}

Este trabalho considerou o modelo para o problema integrado de corte de estoque e dimensionamento de lotes proposto por Poltroniere et al. (2008). Três modelagens matemáticas foram implementadas e resolvidas heuristicamente usando o pacote $\mathrm{Ampl} / \mathrm{Cplex}$ 12.6. Versões relaxadas dos modelos foram, também, resolvidas para obter limitantes inferiores para a solução do problema integrado. As soluções heurísticas obtidas usando o pacote computacional Cplex 12.6 mostraram-se satisfatórias, sendo que, para alguns exemplos, os resultados obtidos foram melhores que os obtidos por Poltroniere et al. (2008). Os resultados mostraram que as relaxações consideradas forneceram bons limitantes inferiores para o problema integrado, com destaque para os obtidos pelo modelo LCGG. Vale ainda destacar que esses limitantes não estão muito distantes das soluções heurísticas obtidas em Poltroniere et al. (2008), o que permitiu observar a qualidade das heurísticas.

Como propostas futuras, pretende-se desenvolver métodos para auxiliar na resolução dos modelos matemáticos de forma exata e/ou heurística via pacote de otimização. Alguns testes iniciais realizados em Poldi e Araujo (2014) mostram que o problema de corte de estoque multiperíodo, que aparece no contexto do problema integrado, pode ser bem resolvido quando o modelo de fluxo em arcos é utilizado. Assim, podemos utilizar estes modelos de forma conjunta com as heurísticas propostas em Poltroniere et al. (2008).

\section{Referências}

[1] M. H. Correia, J. F. Oliveira and J. S. Ferreira, Reel and sheet cutting at a paper mill. Computers \& Operations Research 31, 1223-1243, (2004).

[2] A. A. Farley, Mathematical Programming Models for Cutting-Stock Problems in the Clothing Industry. Operational Research Society 1, 41-53, (1988).

[3] P. C. Gilmore and R. E. Gomory, A linear programming approach to the cutting stock problem. Operations Research 9, 848-859, (1961). 
[4] P. C. Gilmore and R. E. Gomory, A linear programming approach to the cutting stock problem - Part II. Operations Research 11, 863-888, (1963).

[5] P. C. Gilmore and R. E. Gomory, Multi-stage cutting stock problems of two and more dimensions. Operations Research 13, 94-120, (1965).

[6] M.C.N. Gramani and P.M. França, The combined cutting stock and lot-sizing problem in industrial processes. European Journal of Operational Research 174, 509-521, (2006).

[7] M.C.N. Gramani, P.M. França and M. N. Arenales, A Lagrangian relaxation approach to a coupled lot-sizing and cutting stock problem. International Journal of Production Economics 119, 219-227, (2009).

[8] M.C.N. Gramani, P.M. França and M. N. Arenales, A linear optimization approach to the combined production planning model. Journal of the Franklin Institute 348(7), 1523-1536, (2010).

[9] C. T. L. S. Ghidini, Otimização de processos acoplados: programação da produção e corte de estoque. Tese de doutorado. ICMC-USP, São Carlos-SP, (2008).

[10] J. Kallrath, S. Rebennack, J. Kallrath and R. Kusche, Solving Real-World Cutting Stock-Problems in the Paper Industry: Mathematical Approaches, Experience and Challenges, European Journal of Operational Research, (2014). Doi: http://dx.doi.org/10.1016/j.ejor.2014.03.027)

[11] L. Kantorovich, Mathematical methods of organising and planning production (traduzido a partir de um artigo russo, datado de 1939), Management Science 6, 366-422, (1960).

[12] K. C. Poldi and S. A. Araújo, Formulações para o problema de corte de estoque unidimensional multiperíodo. Anais do CNMAC, (2014).

[13] S. C. Poltroniere, K. C. Poldi, F. M. B. Toledo and M.N. Arenales, Coupling Cutting Stock and Lot Sizing Problems in the Paper Industry. Annals of Operations Research 157, 91-104, (2008).

[14] A. Respício and M. E. Captivo, Integrating the cutting stock problem in capacity planning. PhD thesis. Department of Informatics and Centre of Operational Research. University of Lisbon/Portugal, (2002).

[15] S. G. Santos, S. A. Araujo and S. Rangel, Integrated Cutting Machine Programming and Lot Sizing in Furniture Industry. Revista Eletrônica Pesquisa Operacional para o Desenvolvimento 3, 249-266, (2011).

[16] E. Silva, F. Alvelos and J. M. Valério de Carvalho, Integrating two-dimensional cutting stock and lot-sizing problems. Journal of Operational Research Society 65, 108-123, (2014).

[17] F. M. B. Toledo, Dimensionamento de lotes em máquinas paralelas. Tese de Doutorado. DENSIS-UNICAMP, (1998).

[18] W. W. Trigeiro, L. G. Thomas and J. O. McClain, Capacitated lot sizing with setup times. Management Science 35(3), 353-366, (1989).

[19] J. M. Valério de Carvalho, Exact solutin of bin packing problem using column generation and branch-and-bound. Annals of Operations Research 86, 629-659, (1999).

[20] J. M. Valério de Carvalho, LP models for bin packing and cutting stock problems. European Journal of Operational Research 141, 253-273, (2002).

[21] M. Vanzela, S. Rangel, S. A. Araujo and M. A. Carravilla, Um estudo de problema integrado de dimensionamento de lotes e corte de estoque para uma fábrica de móveis de pequeno porte. Anais do XLIV SBPO - Simpósio Brasileiro de Pesquisa Operacional, Rio de Janeiro - RJ, (2012). 